\title{
Aspects of tryptophan metabolism in health and disease: a review
}

\author{
D. P. ROSE
}

From the Alexander Simpson Laboratory for Metabolic Research, St Mary's Hospital Medical School, London W2

In recent years, considerable interest has been developed in the effect of various diseases upon the metabolic pathway by which L-tryptophan is converted to nicotinic acid derivatives. As the list of diseases associated with abnormal tryptophan metabolism has grown, there has been mounting scepticism abciut the specificity and clinical significance of many of the observed changes. The aim of this article is to review some of the more recent developments which have taken place in the study of tryptophan metabolism in man, and to show that many of the reported abnormalities may have as their origin the influence of hormonal and nutritional factors upon the metabolic pathway. Recognition of these factors should help to distinguish between non-specific changes brought about by the metabolic response to the 'stress' of any severe illness, and abnormalities of tryptophan metabolism that are of pathological or clinical significance.

Clinical research into tryptophan metabolism in man has been largely concerned with the levels of intermediate metabolites that are present in urine collected after the patient has received an oral dose of L-tryptophan. The techniques involved have been discussed by Price, Brown, and Yess (1965), and by Musajo and Benassi (1964), who also reviewed the published results obtained in a variety of diseases.

\section{Biochemistry of Tryptophan Metabolism and the Effect of Hormones}

The steps involved in the biosynthesis of nicotinic acid and 5-hydroxytryptamine from L-tryptophan are summarized in Figure 1. Some of these enzymatic reactions require pyridoxal 5-phosphate, the coenzyme derived from the various forms of vitamin $\mathbf{B}_{6}$, as a cofactor. Impaired function of this coenzyme results in an elevated urinary excretion of kynurenine, 3-hydroxykynurenine, and xanthurenic acid, apparently because the kynureninase which is responsible for the conversion of 3-hydroxykynurenine Received for publication 4 May 1971. to 3-hydroxyanthranilic acid is more sensitive to a lack of available coenzyme than are the other vitamin $\mathbf{B}_{6}$-requiring enzymes (Ogasawara, Hagino, and Kotake, 1962). Little is known about the effect of vitamin $\mathbf{B}_{6}$ deficiency upon the human metabolism of amines derived from tryptophan; in the deficient rat there is impaired synthesis of 5-hydroxytryptamine in the liver and kidney, but the activity of brain decarboxylase remains virtually normal (Davis, 1963).

The 3-hydroxylation of kynurenine is catalysed by an enzyme which requires both $\mathrm{NADPH}_{2}$ and a flavin cofactor. Impaired formation of 3-hydroxykynurenine has been demonstrated in riboflavindeficient rats (Charconnet-Harding, Dalgliesh, and Neuberger, 1953; Henderson, Koski, and D'Angeli, 1955), but there do not appear to have been any reports of abnormal tryptophan metabolism in man that may be attributed to riboflavin deficiency.

Tryptophan is an important precursor of nicotinic acid and its derivatives, and loss of this source of the vitamin, due to failure of synthesis by way of the tryptophan-nicotinic acid ribonucleotide pathway, accounts for the pellagra-like skin rash which occurs in three inborn errors of tryptophan metabolism: Hartnup disease (Baron, Dent, Harris, Hart, and Jepson, 1956), hypertryptophanaemia (Tada, Ito, Wada, and Arakawa, 1963), and 3-hydroxykynureninuria (Komrower, Wilson, Clamp, and Westall, 1964). Nicotinic acid derivatives appear to regulate the rate of their own synthesis from L-tryptophan by means of feedback control of the first enzyme of the metabolic pathway, tryptophan oxygenase (Cho-Chung and Pitot, 1967), and failure of this regulatory mechanism is probably responsible for the elevated urinary excretion of tryptophan metabolites which occurs when pellagrins are given an oral dose of the amino acid (Hankes, Leklem, Brown, and Mekel, 1970).

The influence of various hormones upon tryptophan metabolism has been reviewed recently by Rose and McGinty (1970). Tryptophan oxygenase (tryptophan pyrrolase), which catalyses the reaction 


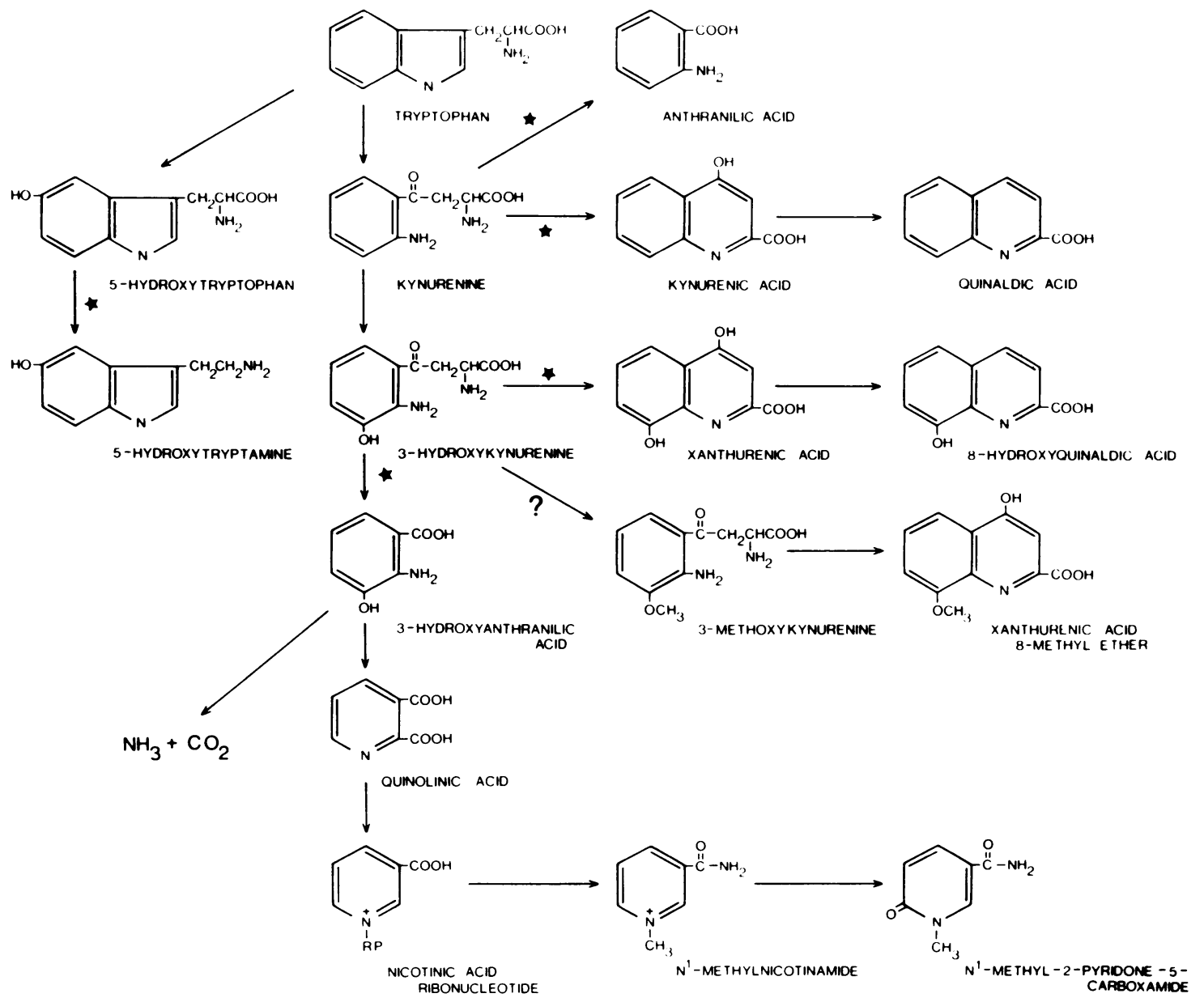

Fig. 1 The metabolic pathway for the biosynthesis of nicotinic acid ribonucleotide and 5-hydroxytryptamine from L-tryptophan and its important side reactions: ${ }^{*} k$ nown pyridoxal 5-phosphate-dependent reactions.

by which the indole ring of L-tryptophan is cleaved to yield formylkynurenine, is regulated by adrenocorticosteroids, oestrogens, and androgens. The activity of this enzyme is elevated by adrenal glucocorticoids (Knox, 1951), and in man hydrocortisone injection results in an increased excretion of kynurenine and other intermediate metabolites in urine collected after an oral dose of L-tryptophan (Altman and Greengard, 1966; Rose and McGinty, 1968). The accumulation of these metabolites seems to occur because there is insufficient pyridoxal phosphate coenzyme available to allow complete metabolism of all of the tryptophan which enters the metabolic pathway, for the increased urinary excretion of metabolites following hydrocortisone injection is prevented by the prior administration of pyridoxine (Rose and McGinty, 1968).
Pregnancy, or the administration of oestrogens, increases tryptophan oxygenase levels in rat liver (Auricchio, Rigillo, and Di Toro, 1960; Rose and Braidman, 1970), and in man, pregnancy, treatment with oestrogens, and the use of oestrogen-containing oral contraceptives all produce grossly elevated urinary excretions of tryptophan metabolites (Brown, Thornton, and Price, 1961; Rose, 1966). The effect of oestrogens upon tryptophan oxygenase appears to be largely an indirect action mediated by way of the adrenal glands, because high enzyme levels do not occur when oestrogens are given to adrenalectomized rats (Braidman and Rose, 1971). In pregnancy there are probably two causes for the abnormal tryptophan metabolism: induction of tryptophan oxygenase by oestrogens, and, later, a true vitamin $\mathbf{B}_{6}$ deficiency which is brought about 
by the foetal demands for the vitamin (Hamfelt and Hahn, 1969).

The administration of synthetic androgens to normal male subjects or women with advanced breast cancer results in a diminished urinary excretion of tryptophan metabolites (McGinty and Rose, 1969; Rose, Randall, and Cramp, 1971). This is probably due to an effect of these steroids upon tryptophan oxygenase, as it has been shown that the treatment of female rats with testosterone reduces the activity of the enzyme (Braidman and Rose, 1971).

From the considerations outlined above it is evident that there are a number of nutritional and hormonal factors which have to be taken into account when making an assessment of the significance of abnormalities of tryptophan metabolism observed in human disease. Special care must be taken to avoid carrying out studies with the object of demonstrating an association between altered tryptophan metabolism and a specific disease while patients are receiving hormones, or when it is likely that adrenocortical secretion is elevated as part of the metabolic response to stress-for example, in the immediate postoperative period or during radiotherapy. Women who are taking oral contraceptives must be excluded from all such investigations.

\section{Vitamin $\mathbf{B}_{6}$ Deficiency}

The effect of an experimentally induced dietary deficiency of vitamin $B_{6}$ upon tryptophan metabolism has been studied in man (Yess, Price, Brown, Swan, and Linkswiler, 1964). Elevated excretions of kynurenine, 3-hydroxykynurenine, and xanthurenic acid occur which are consistent with impaired activity of the enzyme responsible for the conversion of 3-hydroxykynurenine to 3-hydroxyanthranilic acid (Fig. 1). In addition, however, there is an increased excretion of quinolinic acid and this suggests that there may be an unrecognized pyridoxal phosphaterequiring step beyond 3-hydroxyanthranilic acid (Brown, Yess, Price, Linkswiler, Swan, and Hankes, 1965). 3-Hydroxyanthranilic acid excretion was not determined in these investigations, but it is elevated in the urine of oestrogen-treated subjects and returns to normal levels following pyridoxine administration (Rose, 1966).

The kynurenine aminotransferase responsible for the transamination reaction that yields xanthurenic acid from 3-hydroxykynurenine requires pyridoxal phosphate, and although it becomes depleted of the coenzyme at a slower rate than kynureninase, it is conceivable that a sufficiently severe degree of vitamin $\mathbf{B}_{6}$ deficiency may be reached for the rate of this reaction to become impaired, with a consequent reduction in xanthurenic acid synthesis. This has been proposed as the explanation for normal xanthurenic acid, but grossly elevated kynurenine and 3-hydroxykynurenine excretions in the urine of tuberculous patients treated with the vitamin $\mathbf{B}_{6}$ antagunist isoniazid (Price, Brown, and Larson, 1957).

D-Penicillamine is used as a chelating agent in the treatment of Wilson's disease and cystinuria. It has been known for a number of years that both isomers are vitamin $B_{6}$ antagonists (Asatoor, 1964; Jaffe, Altman, and Merryman, 1964), and, in a recent study, Gibbs and Watts (1969) have shown that cystinuric patients treated with $\mathrm{D}$-penicillamine have abnormal tryptophan metabolism which is corrected by pyridoxine. This biochemical evidence of vitamin $\mathbf{B}_{6}$ deficiency is not associated with any clinical manifestations.

With the exception of peripheral neuropathy in isoniazid-treated patients, there is little clear-cut evidence that vitamin $B_{6}$ deficiency is responsible for clinical abnormalities in adult man. However, in infants Coursin (1954) has described a remarkable syndrome of convulsive seizures, behavioural abnormalities, and gross electroencephalographic changes that resulted from the feeding of a vitamin $\mathbf{B}_{6}$-deficient proprietary food preparation.

The expanding concept of 'vitamin $\mathrm{B}_{6}$ dependence' and a discussion of the inborn errors of metabolism such as cystathioninuria and homocystinuria, which show a clinical response to massive doses of pyridoxine, is beyond the scope of this review, and the reader is referred to the report of a recent conference organized by the New York Academy of Sciences (Kelsall, 1969).

\section{Bladder Cancer}

Interest in the study of tryptophan metabolism in man was greatly stimulated when several groups of workers suggested that some of the endogenously produced metabolites of the tryptophan-nicotinic acid pathway are carcinogenic, and are responsible for a proportion of the cases of human bladder cancer in which there is no apparent industrial exposure to chemical carcinogens.

The hypothesis arose when the work of Dunning, Curtis, and Maun (1950) showed that there is a high incidence of bladder tumours in rats fed both 2-acetylaminofluorene and DL-tryptophan, but not when 2-acetylaminofluorene is fed alone. Later, Dyer and Morris (1961) followed up this observation and found that when rats receiving 2-acetylaminofluorene are given supplements of tryptophan they excrete grossly increased amounts of xanthurenic acid, kynurenic acid, and 3-hydroxykynurenine. 
This suggested that the role of 2-acetylaminofluorene is to alter the metabolism of tryptophan by the liver, and that it is this disturbed metabolism which is responsible for the carcinogenesis. The structural similarity between some tryptophan metabolites and known human bladder carcinogens was quickly recognized (Fig. 2).

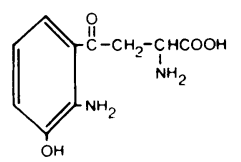

3- HYDROXYKYNURENINE

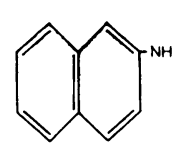

2-NAPHTHYL AMINE

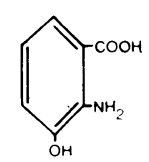

3-HYDROXYANTHRANILIC ACIO

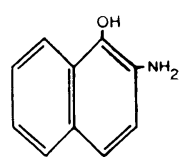

2-AMINO-1-NAPHTHOL
Fig. 2 Structural similarity between 3-hydroxykynurenine and 3-hydroxyanthranilic acid-orthoaminophenolic metabolites of L-tryptophan-and the bladder carcinogen 2-amino-1-naphthol. (2-Amino-1-naphthol is formed in the liver by orthohydroxylation of 2-naphthylamine.)

In 1956, Boyland and Williams reported elevated urinary excretions of 3-hydroxyanthranilic acid, 3-hydroxykynurenine, kynurenine, and anthranilic acid by all of 10 patients studied who were suffering from bladder cancer. Brown and his associates found abnormal tryptophan metabolism in only half of their original series of bladder cancer patients (Brown, Price, Satter, and Wear, 1960), and this incidence has now been confirmed in a second group of patients (Brown, Price, Friedell, and Burney, 1969). In Italy, one group of workers appear to find high urinary excretions of 3-hydroxyanthranilic acid and kynurenine in most of their bladder cancer cases (Alifano, Papa, Tancredi, Elicio, and Quagliariello, 1964), whereas only one third of 201 patients studied by Benassi, Perissinotto, and Allegri (1963) excreted increased quantities of metabolites, the most frequent abnormality being an elevated level of urinary kynurenine.

In parallel with the clinical studies, the pellet implantation technique has been used to determine the carcinogenicity of various tryptophan metabolites for mouse bladder. For these studies a small pellet, consisting of the test compound suspended in cholesterol or some other vehicle, is placed surgically into the bladder lumen. As the pellet is washed with urine the suspected carcinogen is leached out and comes into contact with the bladder mucosa (Jull, 1951).

The technique was first applied to the problem of tryptophan metabolites as bladder carcinogens by Allen, Boyland, Dukes, Horning, and Watson (1957). They obtained a $27 \%$ incidence of tumours in the bladders of mice exposed to 3-hydroxykynurenine or 3-hydroxyanthranilic acid. More recently, the pitfalls of the technique, and the influence upon the results of such factors as the nature of the vehicle used, have been extensively investigated by Bryan and his colleagues (Bryan, Brown, and Price, 1964a and b; Bryan and Springberg, 1966; Bryan, 1969). Their conclusion at the present time is that at least five metabolites-the 8-methyl ether of xanthurenic acid, xanthurenic acid itself, 8-hydroxyquinaldic acid, 3-hydroxykynurenine, and 3-hydroxyanthranilic acid - are mouse bladder carcinogens, as judged by the implantation technique, with the possibility that kynurenine, acetylkynurenine, and quinaldic acid are also active (Bryan, 1969).

Attempts to induce bladder tumours in mice by feeding tryptophan metabolites or injecting them subcutaneously have failed, although 3-hydroxyanthranilic acid produces both leukaemias and lymphoreticular tumours (Bryan, 1968). This failure may be due, in part, to the passage of metabolites to the liver, and their degradation there to inert non-aromatic derivatives. An alternative explanation has been suggested by some of the experiments of Bryan and Springberg (1966). They showed that although the simple subcutaneous injection of the 8-methyl ether of xanthurenic acid will not produce bladder cancers, such tumours do appear if the metabolite is administered by injection to mice that have previously had pure cholesterol pelletsno metabolite present-inserted into their bladders. These results are of considerable interest because they indicate that tryptophan metabolites may act as tumour inducers, and that for neoplastic change to occur a local abnormality within the bladder, such as the presence of a foreign body, is necessary to function as a promoter.

The aetiological significance of abnormal tryptophan metabolism in bladder cancer has been questioned by Benassi et al (1963) on three counts: that normal subjects excrete small quantities of the alleged carcinogens in their urine, that only a minority of their own patients with bladder tumours showed abnormal metabolism of tryptophan, and that elevated urinary levels of tryptophan metabolites 
occur in urological diseases other than bladder cancer, notably renal cell carcinoma.

The first of these objections may be answered by the work of Bryan and Springberg (1966) which was summarized in an earlier paragraph. Vesical schistosomiasis is the clearest example in the human of a situation where abnormal tryptophan metabolism may provide the inducer, and local conditions, in this case the parasite within the bladder mucosa, the foreign body promoter (Abdul-Tawab, Ibrahim, El-Masi, Al-Ghorab, and Makhyoun, 1968). An additional point is that tryptophan metabolites are probably weak carcinogens, and a certain urinary concentration may have to be maintained for the induction of neoplastic change.

Brown et al (1969) have offered a convincing explanation for the discrepancies in the incidence rate of abnormal tryptophan metabolism in bladder cancer, as it has been reported by different groups of workers. They noted that the centres in which a frequent occurrence of such an association has been observed are located in essentially rural areas, such as the State of Wisconsin and southern Italy, whereas Benassi's laboratory is in the northern, more highly industrialized, area of Italy. In view of this, they suggested that in industrial areas there are more cases of bladder cancer resulting from exposure to carcinogens other than tryptophan metabolites, and that these reduce the proportion of the total number of patients studied in whom abnormal tryptophan metabolism is present. In order to test this hypothesis, the levels of urinary tryptophan metabolites in Wisconsin patients were compared with those of cases drawn from the heavily industrialized area of Boston, Massachusetts, and, in agreement with their proposition, it was found that the incidence of abnormal tryptophan metabolism in Boston patients was only $17 \%$ as against $47 \%$ in those from Wisconsin.

Bladder cancer is more common in men than women; the ratio is about 3:2. However, in the study by Brown et al (1969) $75 \%$ of the Wisconsin female patients had abnormal tryptophan metabolism, but only $35 \%$ of the males. The corresponding figures for Boston were $25 \%$ of the women patients and $15 \%$ of the men, but the total number of women was only eight. The observed sex difference suggests that one of the factors which influence the occurrence of elevated excretions of tryptophan metabolites in the urine of bladder cancer patients may be the level of female sex hormones. It is known that women excrete significantly greater amounts of tryptophan metabolites following an oral dose of the aminoacid than men (Michael, Drummond, Doeden, Anderson, and Good, 1964; Rose, 1967a), and that excretion levels are higher at the time of ovulation than immediately after a menstrual period (Rose 1967a), and so an attempt to correlate tryptophan metabolism with hormonal changes in bladder cancer would be of considerable interest.

The increased excretion of tryptophan metabolites following treatment with oestrogens becomes normal when large doses of pyridoxine are administered (Rose, 1966), and so do the high levels of metabolites in the urine of patients with bladder cancer (Brown et al, 1960). In addition to an effect upon kynurenine, 3-hydroxykynurenine, and xanthurenic acid levels, pyridoxine administration produces a reduction in the excretion of 3-hydroxyanthranilic acid by both bladder cancer patients (Durbin and White, 1966) and oestrogen-treated subjects (Rose, 1966). These observations provide, perhaps, further support for the suggestion discussed earlier that there is an unrecognized vitamin- $B_{6}$ dependent reaction beyond 3-hydroxyanthranilic acid formation. In a follow-up study of patients in whom urinary tryptophan metabolite determinations have been made at the time of the initial treatment for bladder cancer, Yoshida, Brown, and Bryan (1970) found that those who developed heterotopic recurrences within five years had all had abnormal metabolism, whereas the tumour-free patients excreted normal urinary metabolite levels. This suggested that the appearance of new tumours, distant from the initial lesion, might be due to the continuing exposure of the bladder mucosa to carcinogenic tryptophan metabolites, in which case treatment with pyridoxine should reduce the recurrence rate.

\section{Other Cancers}

One of the objections to the proposed aetiological role of tryptophan metabolites in carcinoma of the bladder raised by Benassi et al (1963) was that abnormalities of tryptophan metabolism occur in other forms of cancer, notably carcinomas of the prostate and kidney, and Hodgkin's disease. However, these changes, like those seen in breast cancer, may have an endocrine basis. Thus, low levels of androsterone occur in carcinoma of the prostate (Marmorston, Geller, and Weiner, 1969) and, whilst human metastatic renal carcinomas sometimes respond to treatment with androgens (Bloom and Wallace, 1964), in the male golden hamster renal tumours can be produced experimentally by oestrogen administration (Kirkman, 1959).

Approximately half of women with breast cancer excrete elevated amounts of urinary tryptophan metabolites (Rose, 1967a and b; Rose et al, 1970). In the original reports it was suggested that the abnormality is a consequence of increased oestro- 
genic activity, due to either an absolute rise in oestrogen level or an impaired production of androgens, and in support of this it has now been shown that the urinary excretion of aetiocholanolone, one of the principal androgen metabolites, is significantly reduced in breast cancer patients with abnormal tryptophan metabolism (Davis, Leklem, Carlson, and Brown, 1970).

In Hodgkin's disease, the occurrence of abnormal tryptophan metabolism appears to be related to the stage of the disease. Chabner, De Vita, Livingston, and Oliverio (1970) determined both urinary tryptophan metabolites and plasma pyridoxal phosphate levels, and found that abnormalities of both tests were frequently present in the advanced disease, but not in patients who had undergone a complete remission after chemotherapy. Not all of the patients with abnormal tryptophan metabolism had a low plasma pyridoxal phosphate level, and in those who did, treatment with pyridoxine did not return the excretion of metabolites completely to normal.

The occurrence of abnormal tryptophan metabolism in a variety of human cancers and other diseases has raised the suspicion that the changes are nonspecific, and that they are due to stimulation of tryptophan oxygenase activity by the corticosteroids which are released from the adrenal glands as part of the metabolic response to stress (Altman and Greengard, 1966). The changes reported in Hodgkin's disease by Chabner et al (1970) are consistent with this explanation because they occurred in the severely ill patients with advanced disease, and disappeared following successful chemotherapy.

However, certain observations make it unlikely that the abnormalities of tryptophan metabolism which have been described in bladder and breast cancer are due solely to a stress response, although this is probably a contributing factor in some cases. For example, the stress which accompanies the presence of a bladder cancer due to exposure to known industrial chemical carcinogens is presumably of a similar magnitude to that occurring in patients with no such history, and yet abnormal tryptophan metabolism is not seen in industrial cases of bladder cancer (Price and Brown, 1962). And again, the geographical differences in the incidence of disturbed tryptophan metabolism in bladder cancer are not consistent with a non-specific effect of stress.

Bladder cancer patients who have been free of disease for months or years after treatment continue to excrete elevated levels of metabolites, even when thry are studied at home under conditions of minimal stress (Brown et al, 1969). Similarly, in breast cancer about half of those treated by mastectomy and free from recurrence for many months show abnormal tryptophan metabolism (Rose, 1967b), and, finally, the occurrence of abnormal tryptophan metabolism in breast cancer is not dependent upon the stage of the disease; the incidence of abnormalities is the same for untreated patients awaiting surgery, those who have been treated and are apparently free from recurrence months later, and those with recurrent, advanced disease (Rose et al, 1971).

\section{Depression}

It is not intended to review here the considerable amount of evidence which has led to the belief that at least one biochemical abnormality concerned in the development of depressive illness is a defect in the synthesis of 5-hydroxytryptamine from L-tryptophan, although in passing it must be acknowledged that the allocation of a key role to this amine in mood changes is not unanimously accepted (Dewhurst, 1969). Instead, the present discussion will be limited to the interrelationships that are emerging between the level of metabolic activity along the tryptophan-nicotinic acid ribonucleotide pathway and the rate of 5-hydroxytryptamine synthesis.

The metabolism of tryptophan to 5-hydroxytryptamine involves two enzymatic steps (Fig. 1), the first a hydroxylation reaction to yield 5-hydroxytryptophan, and the second a decarboxylation which requires pyridoxal phosphate as a coenzyme (Davis, 1963). Although 5-hydroxytryptamine is synthesized in both liver and kidney, the amine formed at these sites cannot cross the blood-brain barrier (Schanberg, 1963) and so that contained within the brain is produced in situ.

Curzon (1969) has recently summarized the evidence for his hypothesis that low brain 5-hydroxytryptamine concentrations occur in depression because elevated plasma corticosteroid levels induce a high activity of tryptophan oxygenase, which, in turn, increases metabolism along the tryptophannicotinic acid pathway. This hypothesis is applicable to the depression that sometimes develops in women who are using oral contraceptives. Here also, there is abnormal tryptophan metabolism which is probably due to tryptophan oxygenase induction (Rose and Braidman, 1970), and in the rat treatment with a combination of the two contraceptive steroids mestranol and norethynodrel reduces the brain 5-hydroxytryptamine concentration (Nistico and Preziosi, 1970).

An increased rate of turnover of the tryptophannicotinic acid ribonucleotide pathway might conceivably result in reduced 5-hydroxytryptamine production by any of three different mechanisms; preferential utilization of available L-tryptophan 
may result in an inadequate supply of the amine precursor being available; intermediate metabolites may inhibit transport of the aminoacid into the brain tissue; diversion of pyridoxal 5-phosphate to other enzyme systems may impair 5-hydroxytryptophan decarboxylase activity.

The plasma tryptophan level, which presumably reflects the available pool of free aminoacid, does not appear to have been determined in either endogenous depression or women who are using oral contraceptives, but the therapeutic response of depressed patients to large oral doses of L-tryptophan (Coppen, Shaw, Herzberg, and Maggs, 1967) is consistent with there being an inadequate supply for normal 5-hydroxytryptamine synthesis. The second possible mechanism has some experimental support; in the rat kynurenine and 3-hydroxykynurenine decrease the uptake of L-tryptophan into brain slices, and when administered to the live animal these compounds reduce the level of brain 5-hydroxytryptamine (Green and Curzon, 1970). As these metabolites are known to accumulate and to be excreted in excess in the urine of hydrocortisone-treated subjects and those taking oral contraceptives, elevated tryptophan oxygenase activity may impair synthesis of the amine by this mechanism in man. There is no evidence that vitamin $B_{6}$ deficiency will cause inhibition of human brain 5-hydroxytryptophan decarboxylase, and indeed this enzyme is little affected in the vitamin $\mathrm{B}_{6}$ deficient rat, although the liver and kidney enzymes show impaired activity (Davis, 1963). However, there may be a problem of species difference here, because although the decarboxylation step is not the rate-limiting reaction for 5-hydroxytryptamine synthesis in the rat, there is some evidence that it may be in the human (Robins, Robins, Croninger, Moses, Spencer, and Hudgens, 1967).

Not all patients with elevated corticosteroid levels develop depression, even though a tryptophan load test may demonstrate abnormal tryptophan metabolism. Curzon (1969) has suggested that there are compensatory biochemical mechanisms which normally counter any reduction in the level of brain 5-hydroxytryptamine, and that the clinical syndrome of depression appears when these fail. There is some experimental support for such mechanisms, because although a single injection of hydrocortisone causes the amine level to fall in rat brain, the low concentration is not sustained throughout the course of daily steroid injections, but gradually returns to normal (Green and Curzon, 1968). Similarly, the initial reduction in brain 5-hydroxytryptamine when rats are given a combination of mestranol and norethynodrel is not maintained and after about
20 days of daily administration the level has returned to normal (Nistico and Preziosi, 1970).

\section{The Kidney and Tryptophan Metabolism}

In the past it has been generally assumed that abnormalities in the urinary excretion of tryptophan metabolites are due to altered hepatic metabolism of the amino acid. This view has been encouraged because of the key role of tryptophan oxygenase, an enzyme that is only present in liver. However, some recent studies have drawn attention to the kidney as an important site of tryptophan metabolism.

The majority of patients with rheumatoid arthritis excrete increased amounts of tryptophan metabolites in their urine, notably 3-hydroxyanthranilic acid and kynurenine (McMillan, 1960; Spiera, 1966), and this abnormality has been ascribed to high levels of hepatic tryptophan oxygenase (Altman and Greengard, 1966). However, with the development of a method for the determination of serum kynurenine, Spiera and Vallarino (1969) have been able to show that the concentration of this metabolite is reduced in serum from rheumatoid arthritis cases, and that there is a markedly increased renal clearance of kynurenine. Thus, in this disease at least, the kidney plays an important part in the altered urinary excretion of kynurenine, and perhaps the other affected metabolites.

Although tryptophan oxygenase is absent from the kidney, this organ does contain high levels of kynureninase, kynurenine 3-hydroxylase, and kynurenine aminotransferase (De Castro, Brown, and Price, 1957) which may metabolize further the kynurenine produced in the liver. L-kynurenine and 3-hydroxy-L-kynurenine, but not some other metabolites, are actively transported by the small intestinal mucosa (Rose, Yao, and Brown, 1968), and are presumably also subject to renal tubular reabsorption. If kynurenine and 3-hydroxykynurenine are selectively reabsorbed they will be reexposed to both the liver and kidney enzymes, and so any change in reabsorption rate may modify the relative concentrations of the various tryptophan metabolites in urine.

Nothing is known of the effect of hormones upon the handling of tryptophan metabolites by the kidney, but oestrogens inhibit renal kynurenine aminotransferase (Mason, Ford, and Wu, 1969), and this could prove an important topic for future research.

\section{Conclusion}

Despite the intensive research activity of the past 
20 years many of the questions concerning tryptophan metabolism in human disease remain unanswered. It is still not unequivocally established that the elevated urinary excretion of tryptophan metabolites is involved in the aetiology of some human bladder cancers, although the weight of the evidence is becoming irresistible. The possibility that the administration of pyridoxine to correct abnormal tryptophon metabolism will reduce the recurrence rate of bladder tumours is exciting not only in itself, but because it suggests that the early recognition of such a metabolic defect and its correction may actually prevent the onset of some cases of bladder cancer. None of the recent studies have produced a firm indication of the basic defect behind the increased tryptophan metabolite excretions in bladder cancer, and so any comment is of necessity highly speculative. However, the few clues that we have seem to point to an abnormally high requirement for vitamin $B_{6}$ which may be associated with altered endocrine activity.

Specific hormonal changes may also be responsible for the abnormalities of tryptophan metabolism in breast, prostatic, and renal cancers, but much work needs to be done in the way of correlating tryptophan metabolite excretions with the levels of plasma and urinary steroids.

The changes in tryptophan metabolism that occur in women using oral contraceptives are gross, but their clinical significance is completely unknown, and at the present time evidence is being sought for an occult deficiency of vitamin $\mathbf{B}_{6}$. The relationship between the altered tryptophan metabolism and depression complicating the use of oral contraceptives remains to be determined, as does the possibility that it provides a model for the study of the influence of the tryptophan-nicotinic acid ribonucleotide pathway upon brain amine synthesis in endogenous depression.

I wish to thank Miss $\mathbf{J}$. O. Robinson and $\mathrm{Mr} \mathbf{R}$. Strong for their help with the preparation of the manuscript. My own work is su?ported by grants from the Medical Research Council and the Wellcome Trust, and through contract no. Ph-43-67-1344 of the United States National Institutes of Health.

\section{References}

Abdul-Tawab, G. A., Ibrahim, E. K., El-Masi, A., Al-Gharab, M., and Makhyoun, N. (1968). Studies on tryptophan metabolism in bilharzial bladder cancer patients. Invest. Urol., 5, 591-601.

Alifano, A., Papa, S., Tancredi, F., Elicio, M. A., and Quagliariello, E. (1964). Tryptophan-nicotinic acid metabolism in patients with tumours of the bladder and kidney. Brit. J. Cancer, 18, 386-389.

Allen, M. J., Boyland, E., Dukes, C. E., Horning, E. S., and Watson, J. G. (1957). Cancer of the urinary bladder induced in mice with metabolites of aromatic amines and tryptophan. Brit. J. Cancer, 11, 212-228.
Altman, K., and Greengard, O. (1966). Correlation of kynurenine excretion with liver tryptophan pyrrolase levels in disease and after hydrocortisone induction. J. clin. Invest., 45, 1527-1534.

Asatoor, A. M. (1964). Pyridoxine deficiency in the rat produced by D-penicillamine. Nature (Lond.), 203, 1382-1383.

Auricchio, S., Rigillo, N., and Di Toro, R. (1960). Researches on the biosynthesis of nicotinic acid from tryptophan during pregnancy, the foetal and the neonatal periods. I. Tryptophan pyrrolase and 3-hydroxyanthranilic oxidase activity of the rat liver (Italian). Minerva pediat., 12, 1463-1470.

Baron, D. N., Dent, C. E., Harris, H., Hait, E. W., and Jepson, J. B. (1956). Hereditary pellagra-like skin rash with temporary cerebellar ataxia, constant renal amino-aciduria and other bizarre biochemical features. Lancet, 2, 421-428.

Benassi, C. A., Perissinotto, B., and Allegri, G. (1963). The metabolism of tryptophan in patients with bladder cancer and other urological diseases. Clin. chim. Acta, 8, 822-831.

Bloom, H. J. G., and Wallace, D. M. (1964). Hormones and the kidney: possible therapeutic role of testosterone in a patient with regression of metastases from renal adenocarcinoma. Brit. med. J., 2, 476-480.

Boyland, E., and Williams, D.C.(1956). The metabolism of tryptophan. 2. The metabolism of tryptophan in patients suffering from cancer of the bladder. Biochem. J., 64, 578-582.

Braidman, I. P., and Rose, D. P. (1971). The effect of sex hormones on the activity of tryptophan oxygenase and other corticosteroid-inducible enzymes in rat liver. Biochem. J., 122, 28p.

Brown, R. R., Price, J. M., Friedell, G. H., and Burney, S. W. (1969). Tryptophan metabolism in patients with bladder cancer: geographical differences. J. nat. Cancer Inst., 43, 295-301.

Brown, R. R., Price, J. M., Satter, E. J., and Wear, J. B. (1960). The metabolism of tryptophan in patients with bladder cancer. Acta Un. Int. Cancr.. 16, 299-303.

Brown, R. R., Thornton, M. J., and Price, J. M. (1961). The effect of vitamin supplementation on the urinary excretion of tryptophan metabolites by pregnant women. J. clin. Invest., 40, 617-623.

Brown, R. R., Yess, N., Price, J. M., Linkswiler, H., Swan, P., and Hankes, L. V. (1965). Vitamin $\mathbf{B}_{6}$ depletion in man: urinary excretion of quinolinic acid and niacin metabolites. J. Nutr., 87, 419-423.

Bryan, G. T. (1968). Neoplastic response of various tissues to the systemic administration of the 8-methyl ether of xanthurenic acid. Cancer Res., 28, 183-185.

Bryan, G. T. (1969). Pellet implantation studies of carcinogenic compounds. J. nat. Cancer Inst., 43, 255-261.

Bryan, G. T., Brown, R. R., and Price, J. M. (1964a). Incidence of mouse bladder tumors following implantation of paraffin pellets containing certain tryptophan metabolites. Cancer Res., 24, 582-585.

Bryan, G. T., Brown, R. R., and Price, J. M. (1964b). Mouse bladder carcinogenicity of certain tryptophan metabolites and other aromatic nitrogen compounds suspended in cholesterol. Cancer Res., 24, 596-602.

Bryan, G. T., and Springberg, P. D. (1966). Role of the vehicle in the genesis of bladder carcinomas in mice by the pellet implantation technic. Cancer Res., 26, 105-109.

Chabner, B. A., De Vita, V. T., Livingston, D. M., and Oliverio, V. T. (1970). Tryptophan metabolism and plasma pyridoxal phosphate in Hodgkin's disease. New Engl. J. Med., 282, 838-843.

Charconnet-Harding, F., Dalgliesh, C. E., and Neuberger, A. (1953). The relation between riboflavin and tryptophan metabolism. studied in the rat. Biochem. J., 53, 513-521.

Cho-Chung, Y. S., and Pitot, H. C. (1967). Feedback control of rat liver tryptophan pyrrolase. I. End product inhibition of tryptophan pyrrolase activity. J. biol. Chem., 242, 1192-1198.

Coppen, A., Shaw, D. M., Herzberg, B., and Maggs, R. (1967). Tryptophan in the treatment of depression. Lancet, 2, 1178-1180.

Coursin, D. B. (1954). Convulsive seizures in infants with pyridoxinedeficient diet. J. Amer. med. Assc., 154, 406-408.

Curzon, G. (1969). Tryptophan pyrrolase-a biochemical factor in depressive illness? Brit. J. Psychiat., 115, 1367-1374.

Davis, H. L., Leklem, J. E., Carlson, I., and Brown, R. R. (1970). Correlation of urinary steroids and tryptophan and kynurenin metabolism in patients with breast cancer. (Abstr.) Proc. Amer. Ass. Cancer Res., 11, 19.

Davis, V. E. (1963). Effect of cortisone and thyroxine on aromatic amino acid decarboxylation. Endocrinology, 72, 33-38.

De Castro, F. T., Brown, R. R.. and Price, J. M. (1957). The inter- 
mediary metabolism of tryptophan by cat and rat tissue preparations. J. biol. Chem., 228, 777-784.

Dewhurst, W. G. (1969). Amines and abnormal mood. Proc. roy. Soc. Med., 62, 1102-1107.

Dunning, W. F., Curtis, M. R., and Maun, M. E. (1950). The effect of added dietary tryptophan on the occurrence of 2-acetylamino-fluorene-induced liver and bladder cancer in rats. Cancer Res., 10, 454-459.

Durbin, H., and White, R. W. (1966). Penicillamine and pyridoxine in man. Lancet, $1,373$.

Dyer, H. M., and Morris, H. P. (1961). An effect of N-2-fluorenylacetamide on the metabolism of tryptophan in rats. J. nat. Cancer Inst., 26, 315-329.

Gibbs, D. A., and Watts, R. W. E. (1969). Studies on the effect of $\mathrm{D}$-penicillamine and $\mathrm{N}$-acetyl-D-penicillamine on the excretion of some tryptophan metabolites in patients with cystinuria. Clin. Sci., 36. 87-97.

Green, A.R., and Curzon, G.(1968). Decrease of 5-hydroxytryptamine in the brain provoked by hydrocortisone and its prevention by allopurinol. Nature (Lond.), 220, 1095-1097.

Green, A. R., and Curzon, G. (1970). The effect of tryptophan metabolites on brain 5-hydroxytryptamine metabolism. Biochem. Pharmacol., 19, 2061-2068.

Hamfelt, A., and Hahn, L. (1969). Pyridoxal phosphate concentration in plasma and tryptophan load test during pregnancy. Clin. chim. Acta, 25,91-96.

Hankes, L. V., Leklem, J. E., Brown, R. R., and Mekel, R. M. (1970). Abnormal tryptophan metabolism in pellagra. Fed. Proc., 29,884.

Henderson, L. M., Koski, R. E., and D'Angeli, F. (1955). The role of riboflavin and vitamin $\mathrm{B}_{\mathrm{s}}$ in tryptophan metabolism. J. biol. Chem., 215, 369-376.

Jaffe, I. A., Altman, K., and Merryman, P. (1964). The anti-pyridoxine effect of penicillamine in man. J. clin. Invest., 43, 1869-1873.

Jull, J. W. (1951). The induction of tumours of the bladder epithelium in mice by the direct application of a carcinogen. Brit. J. Cancer, $5,328-330$.

Kelsall, M. A., Editor (1969). Vitamin $B_{6}$ in metabolism of the nervous system. Ann. N.Y. Acad. Sci., 166, 1-364.

Kirkman, H. (1959). Estrogen-induced tumors of the kidney. III. Growth characteristics in the Syrian hamster. Nat. Cancer Inst. Monogr., 1, 1-57.

Knox, W. E. (1951). Two mechanisms which increase in vivo the liver tryptophan peroxidase activity: specific enzyme adaptation and stimulation of the pituitary-adrenal system. Brit. J. exp. Path., 32, 462-469.

Komrower, G. M., Wilson, V., Clamp, J. R., and Westall, R. G. (1964). Hydroxykynureninuria. Arch. Dis. Childh., 39, 250-256.

McGinty, F., and Rose, D. P. (1969). Influence of androgens upon tryptophan metabolism in man. Life Sci., 8, 1193-1199.

McMillan, M. (1960). The identification of a fluorescent reducing substance in the urine of patients with rheumatoid arthritis: the excretion of 3-hydroxyanthranilic acid in this and other conditions. J. clin. Path., 13, 140-148.

Marmorston, J., Geller, P. J., and Weiner, J. M. (1969). Pretreatment urinary hormone patterns and survival in patients with breast cancer, prostate cancer, or lung cancer. Ann. N.Y. Acad. Sci., 164, 483-492.

Mason, M., Ford, J., and Wu, H. L. C. (1969). Effects of steroids and nonsteroid metabolites on enzyme conformation and pyridoxal phosphate binding. Ann. N.Y. Acad. Sci., 166, 170-183.

Michael, A. F., Drummond, K. N., Doeden, D., Anderson, J. A., and Good, R. A. (1964). Tryptophan metabolism in man. $J$. clin. Invest., 43, 1730-1746.
Musajo, L., and Benassi, C. A. (1964). Aspects of disorders of the kynurenine pathway of tryptophan metabolism. Advanc. clin. Chem., 7, 63-135.

Nisticò, G., and Preziosi, P. (1970). Contraceptives, brain serotonin, and liver tryptophan pyrrolase. Lancet, 2, 213.

Ogasawara, N., Hagino, Y., and Kotake, Y. (1962). Kynurenine transaminase, kynureninase, and the increase of xanthurenic acid excretion. J. Biochem. (Tokyo), 52, 162-166.

Price, J. M., and Brown, R. R. (1962). Studies on the etiology of carcinoma of the urinary bladder. Acta Un. Int. Cancr., 18, 684-688.

Price, J. M., Brown, R. R., and Larson, F. C. (1957). Quantitative studies on human urinary metabolites as affected by isoniazid and deoxypyridoxine. J. clin. Invest., 36, 1600-1607.

Price, J. M., Brown, R. R., and Yess, N. (1965). Testing the functional capacity of the tryptophan-niacin pathway in man by analysis of urinary metabolites. In Advances in Metabolic Disorders, edited by $R$. Levine and R. Luft, vol. II, pp. 159-224. Academic Press, New York and London.

Robins, E., Robins, J. M., Croninger, A. B., Moses, S. G., Spencer, S. J., and Hudgens, R. W. (1967). The low level of 5-hydroxytryptophan decarboxylase in human brain. Biochem. Med., $1,240-251$.

Rose, D. P. (1966). The influence of oestrogens on tryptophan metabolism in man. Clin. Sci., 31, 265-272.

Rose, D. P. (1967a). The influence of sex, age and breast cancer on tryptophan metabolism. Clin. chim. Acta, 18, 221-225.

Rose, D. P. (1967b). Tryptophan metabolism in carcinoma of the breast. Lancet, 1, 239-241.

Rose, D. P., and Braidman, I. P. (1970). Oral contraceptives, depression and aminoacid metabolism. Lancet, 1, 1117-1118.

Rose, D. P., and McGinty, F. (1968). The influence of adrenocortical hormones and vitamins upon tryptophan metabolism in man. Clin. Sci., 35, 1-9.

Rose, D. P., and McGinty, F. (1970). The effect of steroid hormones on tryptophan metabolism. In Advances in Steroid Biochemistry and Pharmacology, edited by M. H. Briggs, Vol. I, pp. 97-136. Academic Press, London and New York.

Rose, D. P., Randall, Z., and Cramp, D. G. (1971). Tryptophan metabolism by women with breast tumors: effect of age, metastases, and androgen therapy. Submitted for publication.

Rose, D. P., Yao, Y-M. S., and Brown, R. R. (1968). Transport and further metabolism of intermediate compounds of the tryptophan-nicotinic acid ribonucleotide pathway by rat small intestine. Biochim. biophys. Acta. (Amst.), 163, 93-100.

Schanberg, S. M. (1963). A study of the transport of 5-hydroxytryptophan and 5-hydroxytryptamine (serotonin) into brain. $J$. Pharmacol. exp. Ther., 139, 191-200.

Spiera, H. (1966). Excretion of tryptophan metabolites in rheumatoid arthritis. Arthr. and Rheum., 9, 318-324.

Spiera, H., and Vallarino, R. (1969). Serum kynurenine in rheumatoid arthritis. J. clin. Invest., 48, 856-859.

Tada, K., Ito, H., Wada, Y., and Arakawa, T. (1963). Congenital tryptophanuria with dwarfism: H-disease-like clinical features without indicanuria and generalised aminoaciduria. Tohoku J. exp. Med., 80,118-134.

Yess, N., Price, J. M., Brown, R. R., Swan, P. B., and Linkswiler, H. (1964). Vitamin Ba depletion in man: urinary excretion of tryptophan metabolites. J. Nutr., 84, 229-236.

Yoshida, O., Brown, R. R., and Bryan, G. T. (1970). Relationship between tryptophan metabolism and heterotopic recurrences of human urinary bladder tumors. Cancer (Philad.), 25, 773-780. 